\title{
Loeys-Dietz Syndrome Type 1
}

National Cancer Institute

\section{Source}

National Cancer Institute. Loeys-Dietz Syndrome Type 1. NCI Thesaurus. Code C75119.

A rare autosomal dominant syndrome caused by mutations in the TGFBR1 gene. It is characterized by vascular abnormalities (aortic and arterial aneurysms, aortic dissection, and tortuosity of the arteries), hypertelorism, bifid uvula, and early fusion of the skull bones. 